\title{
Evidence for a Neutral Grain-Boundary Barrier in Chalcopyrites
}

\author{
Susanne Siebentritt, ${ }^{1, *}$ Sascha Sadewasser, ${ }^{2}$ Mark Wimmer, ${ }^{2}$ Caspar Leendertz, ${ }^{2}$ Tobias Eisenbarth, ${ }^{2}$ and \\ Martha Ch. Lux-Steiner ${ }^{1}$ \\ ${ }^{1}$ Department of Solar Energy, Hahn-Meitner Institut Berlin, Glienicker Straße 100, 14109 Berlin, Germany \\ and Freie Universität Berlin, Fachbereich Physik Arnimallee 14, 14195 Berlin, Germany \\ ${ }^{2}$ Department of Solar Energy, Hahn-Meitner Institut Berlin, Glienicker Straße 100, 14109 Berlin, Germany
}

(Received 27 June 2006; published 2 October 2006)

\begin{abstract}
Single grain boundaries in $\mathrm{CuGaSe}_{2}$ have been grown epitaxially. Hall measurements indicate a barrier of $30-40 \mathrm{meV}$ to majority carrier transport. Nevertheless, local surface potential measurements show the absence of space charge around the grain boundary; i.e., it is neutral. Theoretical calculations [Persson and Zunger, Phys. Rev. Lett. 91, 266401 (2003)] have predicted a neutral barrier for the present $\Sigma 3$ grain boundary. Thus, we have experimentally shown the existence of a neutral grain-boundary barrier, however, smaller than theoretically predicted.
\end{abstract}

PACS numbers: 72.80.Ey, 61.72.Mm, 71.55.Gs, 72.20.-i

The electronic structure of $\mathrm{Cu}$ chalcopyrites shows numerous peculiarities, including, i.e., their grain-boundary properties. The first model [1,2] for the electronic structure of grain boundaries in chalcopyrites was based on the Seto model [3], where charged defects at grain boundaries cause a barrier for majority carriers (holes in the chalcopyrites discussed here). Barriers and space charge regions have been found in polycrystalline films by Hall measurements and Kelvin probe force microscopy (KPFM) (see, e.g., $[1,4-6])$. Theoretically it has been predicted that grain boundaries in chalcopyrites represent a barrier without charged defects $[7,8]$. In the $\mathrm{Cu}$ chalcopyrites, the top of the valence band is formed from antibonding $\mathrm{Cu} d$ states, which lifts it to higher energy compared to the corresponding II-VI compounds [9]. Grain boundaries consisting of $\{112\}_{\text {tet }}$ planes in the tetragonal system [10], corresponding to the $\{111\}_{\text {cub }}$ planes in the cubic system, are proposed to be $\mathrm{Cu}$ deficient, which results in a lowering of the valence band maximum. This results in a barrier in the valence band without a space charge at the grain boundary. Recently it has been argued that only a specific structure of a $\{112\}_{\text {tet }}$ grain boundary leads to this barrier and that the experimentally observed structures should not show a neutral barrier [11].

Grain boundaries along the $\{112\}_{\text {tet }}$ planes can be described as twins. The coincidence site lattice (CSL) of such grain boundaries is characterized by a $\Sigma$ value of 3 , the lowest $\Sigma$ value possible. Thus, it is expected that grain boundaries along $\{112\}_{\text {tet }}$ planes show a low defect density. A generating function of the CSL for the cubic system has been derived in Ref. [12]. Since the tetragonal distortion of chalcopyrites is small and since the CSL concerns only the two-dimensional plane of the grain boundary we assume the generating function for the cubic system as an approximation for the chalcopyrite system. Then it becomes clear that a polycrystalline film with $\{220\}_{\text {tet }}$ (i.e., $\{110\}_{\text {cub }}$ ) texture and vertical grain boundaries contains predominantly $\Sigma 3$ grain boundaries. In fact, it has been shown that polycrystalline absorber films with $\{220\}_{\text {tet }}$ texture result in higher efficiencies of the corresponding solar cells compared to the usual $\{112\}_{\text {tet }}$ texture [13]. Therefore, $\Sigma 3$ grain boundaries are the ones appearing in the most successful solar cells and are the ones predicted to show a neutral barrier. So far, no experimental evidence for the neutral barrier grain boundaries exists. A recent Letter [14] indicates a $\mathrm{Cu}$ deficiency at grain boundaries; however, work function measurements by the secondary electron threshold method in fact show evidence of a decreased work function, i.e., a space charge around the grain boundaries, as was found by the previous KPFM measurements.

In this Letter we investigate a single $\Sigma 3$ grain boundary of $\mathrm{CuGaSe}_{2}$ grown epitaxially by metal organic vapor phase epitaxy (MOVPE) on a GaAs wafer. The wafer consists of two grains showing a (001) cub and a (221) cub surface, connected via a $\Sigma 3$ grain boundary. The film grows epitaxially on both sides, as indicated by electron backscatter diffraction. The grain boundary in the film is a direct continuation of the grain boundary in the wafer and shows also a twin structure as shown by transmission electron microscopy (TEM) investigations. The film orientation is $(001)_{\text {cub }}$ on the $(001)_{\mathrm{cub}}$-GaAs side showing the usual smooth surface, and (221) cub on the other side of the grain boundary, which breaks up into $\{112\}_{\text {tet }}$ facets, as has been observed previously [15]. All films are grown under $\mathrm{Cu}$ excess, i.e., stoichiometric chalcopyrite, which accommodates the $\mathrm{Cu}$ excess in $\mathrm{Cu}_{x} \mathrm{Se}$ layer on top of the film which is easily etched away in a cyanide solution. Further details of the growth process and the structure of the grain boundary are presented in a separate paper [16]. The electronic properties of the grain boundary are investigated by KPFM and by Hall measurements. KPFM measurements probe the electrostatic field of the grain boundary, while Hall measurements probe the barrier for majority charge carriers, which becomes evident as an activated behavior of the temperature dependence of the mobility. 
Our KPFM is based on an ultrahigh vacuum (UHV) noncontact atomic force microscope, which uses the second harmonic of the cantilever for the Kelvin probe measurement of the work function and thus allows for a simultaneous measurement of the topography and the work function with high spatial and energy resolution [17]. The work function is calibrated using highly oriented pyrolytic graphite as a reference. After KCN etching, the sample was transferred through ambient air into the UHV system and sputter cleaned to remove surface contaminations before the KPFM measurement [18]. For Hall measurements the samples are broken into three pieces: two square pieces for the bulk measurement on each side of the grain boundary, measured by the van der Pauw method, and one piece containing the grain boundary with stripe contacts for conductivity measurement across the grain boundary by the four point method. The contact distance on this piece is $3 \mathrm{~mm}$ with the grain boundary in the middle. The van der Pauw measurement of the two bulk pieces yields the charge carrier concentration and the mobility for the bulk of the two sides of the bicrystal.

The temperature dependence of the conductivity of the middle piece (triangles) containing the grain boundary is shown in Fig. 1(a) in comparison with the conductivity of the bulk (solid line). The grain-boundary conductivity is hardly different from the bulk conductivity; a slightly steeper slope cannot be excluded. To obtain a more detailed insight, the conductivity of the middle piece with the grain boundary is modeled by a series connection of the resistances of the bulk part on either side of the grain boundary and of the grain boundary itself: $R=R_{(001)}+$ $R_{\mathrm{GB}}+R_{(221)}$. The resistance of the bulk parts is calculated from the measured dimensions of the sample, the distance of the contacts from the grain boundary, and the conductivity obtained from the van der Pauw measurements of the bulk pieces. Thus, the conductivity of the grain boundary itself is determined. From the conductivity and the average of the carrier concentration of the two bulk pieces the mobility across the grain boundary is calculated. The temperature dependence of the mobility of the grain boundary is presented in Fig. 1(b). It shows the same general behavior as the mobility in polycrystalline films [1,2]: almost constant around room temperature, followed by an activated behavior and deviation from the activated behavior at lower temperatures which is attributed to transport via defects. Since this low temperature deviation from the activated behavior is attributed to defect related transport, those last two points were not included in the linear extrapolation. The activation energy in this case is $32 \mathrm{meV}$. Barriers with activation energies between 20 and $40 \mathrm{meV}$ have been found for various samples, with a trend to higher activation energies for samples grown under higher $\mathrm{Cu}$ excess.

In polycrytsalline films the barriers were assigned to charged grain-boundary defects [1]. To investigate the

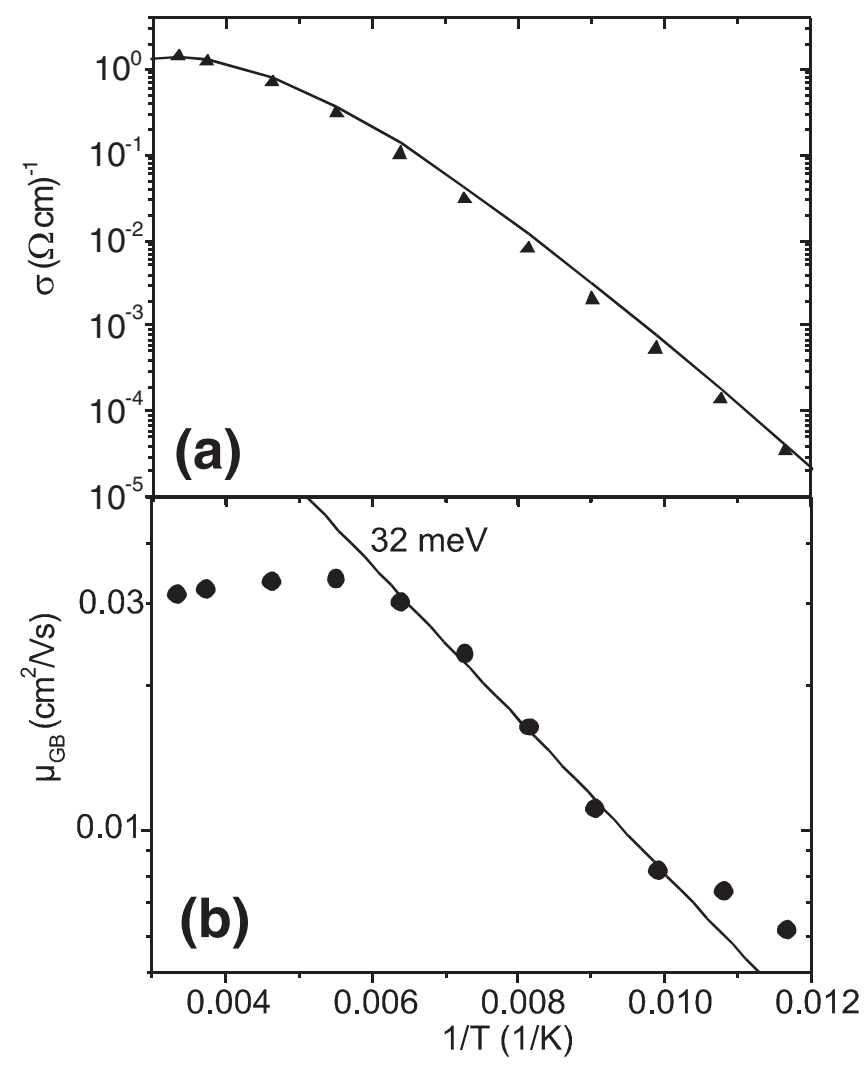

FIG. 1. (a) Temperature dependent conductivity of a $3 \mathrm{~mm}$ wide piece of the film containing the grain boundary $(\mathbf{\Delta})$ together with the conductivity of the bulk (solid line) and (b) temperature dependence of the mobility of the grain boundary itself showing an activated behavior in an intermediate temperature range. Error bars are of the size of or smaller than the dots.

presence of charges, we performed KPFM measurements on the present $\Sigma 3$ grain boundary. The topography of the $\mathrm{CuGaSe}_{2}$ surface around the grain boundary is shown in Fig. 2(a) as a 3D representation and the simultaneously measured work function in Fig. 2(b). The left-hand side shows the smooth $(001)_{\text {cub }}$ surface with the well-known trenches forming along the [110] direction [15]. The righthand side shows the onset of the faceting into (112) tet faces which occurs on the (221) cub side of the grain boundary. The work function varies for the different surface orientations, as was observed before [19]. No dip in the work function is seen along the grain boundary, in contrary to the observation in most polycrystalline films where a work function dip is in fact observed along the grain boundaries $[4,6,20]$. For a more detailed investigation a line profile of the work function across the grain boundary together with the topography line profile is shown in Fig. 3. The topography scan shows left of $1.1 \mu \mathrm{m}$ the smooth $(001)_{\text {cub }}$ surface and then the slope towards a (112) tet facet indicative of the (221) cub surface. A constant work function of $5.16 \mathrm{eV}$ is detected on the $(001)_{\text {tet }}$ side, followed by an increase at the grain boundary to a value of $5.20 \mathrm{eV}$ on the 

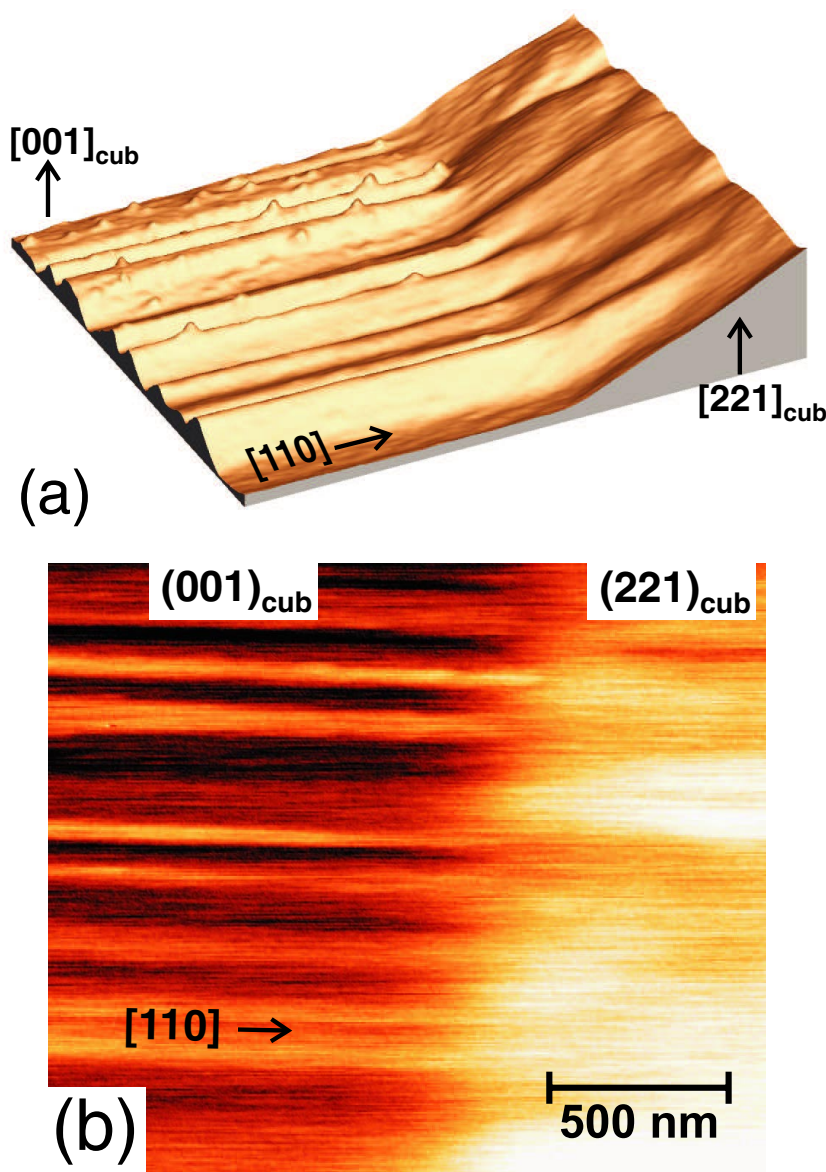

FIG. 2 (color online). 3D representation of the topography (a) and work function (b) close to the epitaxially grown $\Sigma 3$ grain boundary, as measured by KPFM. Both images are $2 \mu \mathrm{m} \times$ $1.7 \mu \mathrm{m}$. The vertical scale in the topography is $\approx 110 \mathrm{~nm}$ and the variations in the work function image are $\approx 40 \mathrm{meV}$.

$(112)_{\text {tet }}$ facets, in accordance with previous observations [19]. No dip or bump beyond the noise level of $\approx 5 \mathrm{meV}$ is visible at the grain boundary, which would be expected for a positively or negatively charged grain boundary, respectively.

To check if the small barrier of $30-40 \mathrm{meV}$ could be missed in the KPFM measurement we have simulated the KPFM line profile across the grain boundary with a transition between the two observed work function values plus a dip in the work function corresponding to a $30 \mathrm{meV}$ barrier. The space charge region width for such a barrier and the doping concentration of a few $10^{16} \mathrm{~cm}^{-3}$, as obtained from the Hall measurements, is calculated to $\approx 50 \mathrm{~nm}$. The averaging influence of the tip geometry on the measurement of the surface potential in KPFM is considered by finite element simulations of the electrostatic field distribution [21]. This results in a broadened dip as seen in Fig. 4, which would easily be resolved in our KPFM measurements. Similar simulations with a smaller dip lead to the conclusion that even a $10-15 \mathrm{meV}$ barrier due to charged defects could be resolved experimentally.

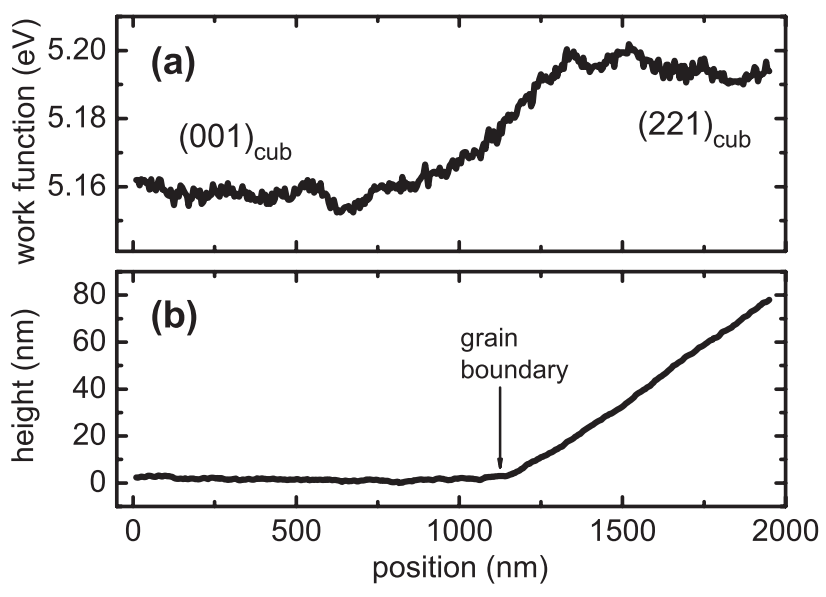

FIG. 3. KPFM line profile of the work function (a) and topography (b) across the grain boundary. The KPFM scan shows no indication of a space charge at the grain boundary.

Thus, we conclude that the major contribution to the barrier observed in the Hall measurements is due to a neutral valence band offset.

Therefore, $\Sigma 3$ grain boundaries in $\mathrm{CuGaSe}_{2}$ show a barrier to majority carrier transport, evident from Hall measurements, without the presence of charges as seen from the KPFM measurements. Thus, we have experimentally observed a neutral barrier, as was predicted by $a b$ initio calculations $[7,8]$. This barrier resulting from the lowering of the valence band maximum caused by the $\mathrm{Cu}$ deficiency of the grain boundary was predicted to be $550 \mathrm{meV}$ in $\mathrm{CuGaSe}_{2}$ [8]. Since all our films are grown under $\mathrm{Cu}$ excess it is possible that our grain boundaries are less $\mathrm{Cu}$ deficient than the equilibrium grain boundaries calculated and that therefore the lowering of the valence band maximum is smaller than in the equilibrium case.

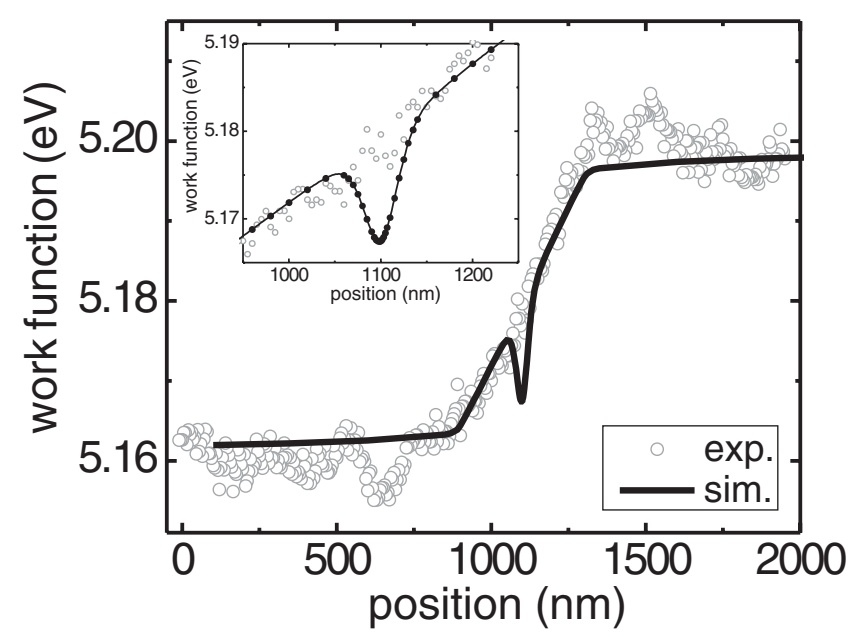

FIG. 4. Simulation of the KPFM line profile (solid line) across the grain boundary assuming a $30 \mathrm{meV}$ barrier at the grain boundary. The points show the comparison with the experimental data. 
The authors would like to thank J. Albert for the MOVPE growth, C. Kelch and M. Kirsch for further sample preparation, A. Rockett for numerous discussions, and the German Research Foundation (DFG) and the German Research Ministry (BMBF) for funding.

*Electronic address: siebentritt@hmi.de

[1] S. Schuler, S. Nishiwaki, J. Beckmann, N. Rega, S. Brehme, S. Siebentritt, and M.Ch. Lux-Steiner, in 29th IEEE Photovoltaic Specialist Conference (IEEE, New York, 2002), p. 504.

[2] S. Siebentritt and S. Schuler, J. Phys. Chem. Solids 64, 1621 (2003).

[3] J. Y. W. Seto, J. Appl. Phys. 46, 5247 (1975).

[4] S. Sadewasser, Th. Glatzel, S. Schuler, S. Nishiwaki, R. Kaigawa, and M.Ch. Lux-Steiner, Thin Solid Films 431-432, 257 (2003).

[5] C.-S. Jiang, R. Noufi, J. A. AbuShama, K. Ramanathan, H. R. Moutinho, J. Pankow, and M. M. Al-Jassim, Appl. Phys. Lett. 84, 3477 (2004).

[6] D. Fuertes Marrón, S. Sadewasser, A. Meeder, Th. Glatzel, and M. Ch. Lux-Steiner, Phys. Rev. B 71, 033306 (2005).

[7] C. Persson and A. Zunger, Phys. Rev. Lett. 91, 266401 (2003).

[8] C. Persson and A. Zunger, Appl. Phys. Lett. 87, 211904 (2005).

[9] J. E. Jaffe and A. Zunger, Phys. Rev. B 28, 5822 (1983).
[10] By the subscript "tet" we denote directions and planes in the tetragonal system and by "cub" the same in the cubic system.

[11] Y. Yan, R. Noufi, and M. M. Al Jassim, Phys. Rev. Lett. 96, 205501 (2006).

[12] H. Grimmer, Acta Crystallogr. Sect. A 40, 108 (1984).

[13] M. Contreras, B. Egaas, K. Ramanathan, J. Hiltner, F. Hasoon, and R. Noufi, Progress in Photovoltaics: Research and Applications 7, 311 (1999).

[14] M. J. Hetzer, Y.M. Strzhemechny, M. Gao, M. A. Contreras, A. Zunger, and L.J. Brillson, Appl. Phys. Lett. 86, 162105 (2005).

[15] S. Siebentritt, N. Papathanasiou, J. Albert, and M. Ch. Lux-Steiner, Appl. Phys. Lett. 88, 151919 (2006).

[16] S. Siebentritt, T. Eisenbarth, A. Rockett, J. Albert, P. Schubert-Bischoff, and M. Ch. Lux-Steiner, J. Phys. Condens. Matter (to be published).

[17] Ch. Sommerhalter, Th. W. Matthes, Th. Glatzel, A. JägerWaldau, and M. Ch. Lux Steiner, Appl. Phys. Lett. 75, 286 (1999).

[18] S. Sadewasser, in Wide-Gap Chalcopyrites, edited by S. Siebentritt and U. Rau (Springer, New York, 2006), p. 193.

[19] S. Sadewasser, T. Glatzel, M. Rusu, A. Jäger-Waldau, and M. Ch. Lux-Steiner, Appl. Phys. Lett. 80, 2979 (2002).

[20] G. Hanna, Th. Glatzel, S. Sadewasser, N. Ott, H. P. Strunk, U. Rau, and J.H. Werner, Appl. Phys. A 82, 1 (2006).

[21] U. Zerweck, C. Loppacher, T. Otto, S. Grafström, and L. M. Eng, Phys. Rev. B 71, 125424 (2005). 